\title{
Economic Impact of Early-in-Hospital Diagnosis and Initiation of Eculizumab in Atypical Haemolytic Uraemic Syndrome
}

\author{
Michael Ryan ${ }^{1} \cdot$ Bonnie M. K. Donato ${ }^{2} \cdot$ William Irish $^{3} \cdot$ Christoph Gasteyger $^{4} \cdot$ Gilbert L'Italien $^{2} \cdot$ Jeffrey Laurence $^{5}$
}

Published online: 12 December 2019

(c) The Author(s) 2019

\begin{abstract}
Background Atypical haemolytic uraemic syndrome (aHUS) is a rare, potentially life-threatening condition caused by dysregulation of the complement pathway. Eculizumab is currently the only approved treatment for this disorder.

Objective Our objective was to investigate the impact of early administration of eculizumab on inpatient resource use and hospitalisation costs in 222 patients with aHUS.

Methods We conducted a retrospective analysis of the Premier Perspective ${ }^{\circledR}$ Hospital Database, including patients with a diagnosis of aHUS and evidence of eculizumab use for aHUS. Early initiation was defined as having received eculizumab within 7 days of admission, with late initiation defined as starting eculizumab on day 8 or later. This date represents the average time required to obtain a specific diagnostic test to discriminate aHUS from a similar haemolytic syndrome that requires a different treatment. Outcome measures were time from first eculizumab initiation to discharge, discharge status or death, days spent in the intensive care unit (ICU), readmission indicators, dialysis indicators, and total hospital costs. Time from first eculizumab initiation to discharge was analysed using a generalised linear model with a log link and an assumed underlying negative binomial distribution. Logistic regression models were used to test the statistical significance of early versus late initiation as a predictor of the occurrence of readmissions, dialysis, and death. Total hospital costs were analysed using a generalised linear model with a log link and an assumed underlying gamma distribution.

Results Before modelling, total length of stay and ICU duration were significantly longer for late initiators than for early initiators, and significantly more late initiators were readmitted within 90 days. Late initiation was associated with significantly higher hospital costs than early initiation. After multivariable analysis, late initiators were 3.2 times more likely to require dialysis. However, there was no significant association between early initiation and time to discharge, readmission, or death for any definition or early initiation after multivariable analysis. Estimated total hospital costs (year 2017 values) were \$US103,557 in late initiators and \$US85,776 in early initiators $(p=0.0024)$.

Conclusion Initiation of eculizumab within 7 days of hospitalisation is associated with lower dialysis rates, less time in ICU, less plasmapheresis, and lower hospitalisation costs compared with late initiation.
\end{abstract}

Data in this manuscript were previously presented at the 60th American Society of Hematology Annual Meeting, 1-4 December 2018, San Diego, CA, USA (abstract \#3565).

Electronic supplementary material The online version of this article (https://doi.org/10.1007/s40273-019-00862-w) contains supplementary material, which is available to authorized users.

Michael Ryan

mryan@ctifacts.com

1 CTI Clinical Trial and Consulting Services Inc., 100 E. RiverCenter Blvd, Covington, KY 41011, USA

2 Boehringer-Ingelheim Pharmaceutical Inc., Ridgefield, CT, USA
3 Brody School of Medicine at East Carolina University, Greenville, NC, USA

4 Alexion Pharma GmbH, Zurich, Switzerland

5 New York Presbyterian Hospital and Weill Cornell Medical College, New York, NY, USA 


\section{Key Points for Decision Makers}

Patients with atypical haemolytic uraemic syndrome (aHUS) for whom eculizumab treatment is delayed beyond 7 days are significantly more likely to require dialysis than those who start treatment earlier.

Delaying eculizumab initiation beyond 7 days also leads to significantly increased healthcare costs compared with earlier initiation.

\section{Introduction}

Thrombotic microangiopathies (TMAs) are a group of disorders characterised by thrombocytopenia and microangiopathic haemolytic anaemia [1]. The major TMAs include disseminated intravascular coagulation, thrombotic thrombocytopenic purpura (TTP), Shiga toxin-associated haemolytic uraemic syndrome (STEC-HUS), and atypical haemolytic uraemic syndrome (aHUS). These conditions can lead to ischaemic organ injury and dysfunction in numerous organs, including the brain, kidneys, heart, pancreas, liver, lungs, eyes, and skin. TTP and aHUS are clinically very similar in presentation but have very different aetiologies. TTP is caused by a congenital or acquired deficiency of an enzyme known as ADAMTS13 (a disintegrin and metalloprotease with thrombospondin type 1 repeats-13). Plasma exchange is the first line of treatment for TTP and can be life saving. In contrast, aHUS is a rare and potentially lifethreatening condition associated with TMA that results from dysregulation of the complement pathway, primarily affecting the kidneys $[1,2]$. Patients with aHUS or TTP often present in the intensive care unit (ICU) for urgent specialist care. Historically, treatment for aHUS, as in TTP, involved plasma infusion or exchange, although, in stark contrast to TTP, there is little evidence for its efficacy [2].

Although plasma exchange elicits a treatment response in approximately $70 \%$ of patients with aHUS [3], outcomes are still very poor. In a French cohort, $29 \%$ of paediatric and $56 \%$ of adult patients either died or required renal replacement therapy within 1 year of disease manifestation [4]. In an Italian cohort, the 3-year proportion of patients with endstage renal disease or death was $48 \%$ and $67 \%$ in paediatric and adult patients, respectively [3]. Outcomes in patients with identified pathogenic variants tend to be worse.

Eculizumab is a humanised monoclonal antibody that binds to complement component $\mathrm{C} 5$, blocking its cleavage into $\mathrm{C} 5 \mathrm{a}$ and $\mathrm{C} 5 \mathrm{~b}$ and preventing the formation of the terminal complement complex C5b-9, thought to be responsible for the endothelial damage and platelet activation underlying the pathology of aHUS [5]. A recent in vivo study in complement factor $\mathrm{H}$ mice implicated specific involvement of C5b-9 in driving the development of renal TMA [6]. At the time of this analysis, eculizumab was the only pharmacological treatment approved by the US FDA and the European Medicines Agency (EMA) for aHUS (recently, the FDA approved ravulizumab, a long-acting C5 inhibitor engineered from eculizumab, for aHUS). In clinical trials, eculizumab maintained haematological normalisation in over $80 \%$ of patients, demonstrating significant improvement in renal function (measured by estimated glomerular filtration rate $[\mathrm{eGFR}])[7,8]$.

In a recent post hoc analysis of four phase II, open-label, single-arm, prospective clinical studies of eculizumab in aHUS, initiation of treatment within 7 days after manifestation of TMA symptoms was associated with significantly greater improvement in eGFR compared with later initiation [9]. Day 7 was chosen as the cut-off as it takes 3-7 days to obtain the results of the ADAMTS13 activity assay in most hospitals in the USA and Europe [10,11]. After 1 year, mean change in eGFR from baseline was $57 \mathrm{~mL} / \mathrm{min} / 1.73 \mathrm{~m}^{2}$ in patients treated within 7 days compared with $23 \mathrm{~mL} / \mathrm{min} /$ $\mathrm{m}^{2}$ in those treated after 7 days $(p=0.0098)$. A sustained increase in eGFR after 1 year was observed in $81 \%$ and $47 \%$ of patients, respectively [9]. However, difficulties in reaching a diagnosis of aHUS result in delayed initiation of therapy and an associated impact on clinical outcomes, including mortality [12]. There is no definitive test for aHUS. Diagnosis is made by exclusion of other TMAs, particularly the exclusion of TTP using an assay to detect reduced activity of ADAMTS13, and STEC-HUS using assays for Shiga toxin resulting from Shiga-toxin-producing Escherichia coli infection [1,2].

Few studies have investigated the efficacy of eculizumab therapy from a real-world perspective [13, 14]. Based on results from these phase II studies [9], we hypothesised that patients initiating eculizumab treatment on day 8 of their hospital stay or later would have greater consumption of inpatient resources and increased hospital costs than those who initiate therapy earlier. The objective of the present study was to evaluate the impact of early ( $<8$ days after hospital admission) versus late initiation of eculizumab for treatment of an aHUS form of TMA in hospitalised patients.

\section{Methods}

\subsection{Study Design}

This was a retrospective multivariable analysis using 'real-world' data from the Premier Perspective ${ }^{\circledR}$ Hospital 
Database. This database contains data from over 350 million patient encounters (one-fifth of hospital discharges in the USA) including data from standard hospital discharge files, including a patient's demographic and disease state, and information on billed services, including medications, laboratory, diagnostics and therapeutic services in de-identified patient daily service records. Information on hospital characteristics, including geographic location, bed size and teaching status are also available. All resource usage and cost data used in this study were obtained from the Premier Perspective ${ }^{\circledR}$ Hospital Database. All data used to perform this analysis were de-identified and accessed in compliance with the Health Insurance Portability and Accountability Act. Medical charts and treatment decisions were not specifically reviewed.

\subsection{Patients}

Patients included in the analysis had inpatient hospitalisation recorded in the Premier Perspective ${ }^{\circledR}$ Hospital Database between October 2011 and March 2016 with (1) a diagnosis code for HUS (International Classification of Diseases, Ninth Revision, Clinical Modification [ICD-9-CM] 283.11 or ICD-10 D59.3) and/or TMA (ICD-9, 446.6; ICD-10, M31.1) and (2) evidence of eculizumab use in this context while hospitalised, as there is no separate diagnostic code for aHUS. Unlike TTP, marked renal disease is a characteristic manifestation of aHUS. Records of patients without one of these conditions were reviewed manually by one of the authors (a clinical expert haematologist) who has published extensively on the diagnosis and treatment of thrombotic microangiopathies, including aHUS and TTP to corroborate the probable diagnosis of aHUS in the eculizumab-treated individual.

Patients who received eculizumab in an outpatient setting within the 6 months preceding the index hospitalisation (washout period) were excluded, as were those with evidence of current or previous bone marrow transplant or HIV/AIDS and STEC-positive patients.

\subsection{Variables of Interest}

The reference timepoint was day of hospital admission. The independent variable in the analysis was time of eculizumab initiation. Based on a pooled analysis of eculizumab clinical trial data [9], early initiation was defined as having received eculizumab within 7 days of admission, with late initiation defined as starting eculizumab on day 8 or later. Patients were grouped as early or late initiators of eculizumab after the diagnosis of aHUS had been confirmed. The model identified dependent variables that could impact on time of eculizumab initiation. To understand factors affecting initiation, the role of disease severity and key patient characteristics was evaluated. These variables were decided based on disease relevance and clinical importance. Pre-defined confounders used in modelling were patient characteristics (age, sex, race), hospital characteristics (urban/rural, teaching status) and comorbidities (Elixhauser score). Chronic conditions were defined using the Elixhauser Comorbidity Index (ECI), which is based on 31 categories of comorbidities associated with mortality (e.g. congestive heart failure, hypertension, diabetes, renal failure, liver disease, metastatic cancer). A composite ECI score has a potential range of 0-31 [15]. When modelling total costs, length of stay (including time in ICU) was included because of the inherent bias in the definitions of early and late initiation. As late initiators of eculizumab treatment have an inherently longer length of stay (as they are in hospital for 8 days before being considered a late initiator), length of stay was controlled for when modelling costs.

Outcome measures in the analysis were related to resource utilisation and included time (in days) from first eculizumab initiation to discharge, discharge status of in-hospital death, days spent in ICU, readmission indicators (readmission was defined as admission after being discharged), dialysis indicators (identified by procedure code), and total hospital costs (\$US, year 2017 values). Univariate comparison for continuous variables was conducted using a $t$ test and for categorical variables, a chi-squared test.

\subsection{Multivariable Analysis}

Time from first eculizumab initiation to discharge was analysed using a generalised linear model with a log link and an assumed underlying negative binomial distribution. Logistic regression models were used to test the statistical significance of early versus late initiation as a predictor of the occurrence of readmissions, dialysis and death. Total hospital costs were analysed using a generalised linear model with a log link and an assumed underlying gamma distribution. In all the varying definitions of early versus late, modified Park tests were run to test whether the power of the mean $(\ln (\mathrm{p}))$ was different than 2 , which would imply the gamma distribution was not appropriate. In Table 1 in the Electronic Supplementary Material (ESM), all estimates are between 2.5 and 2.7, with the confidence bounds including 2. From these results, we assumed the gamma distribution would be appropriate for these models. We conducted a sensitivity analysis in which the definition of early initiation of eculizumab was varied from 3 to 10 days and the day of initiation of eculizumab was modelled as a continuous variable. Least squares mean estimates were generated for multivariable estimates of total costs. 


\section{Results}

In total, 222 patients meeting the clinical inclusion criteria were included in the analysis (Fig. 1). Of these, 72 (32.4\%) were early initiators and $150(67.6 \%)$ were late initiators. Demographic characteristics of the included patients are shown in Table 1.

Univariable analysis of hospital visit characteristics for early and late initiators are shown in Table 2. Univariate comparison for continuous variables was conducted using a t-test and, for categorical variables, a chi-squared test. The total hospital length of stay and ICU duration were significantly longer for late initiators than for early initiators (mean 29.0 vs. 16.4 days [ $p<0.001$ ] and 12.4 vs. 8.9 days $[p=0.047]$, respectively), and significantly more

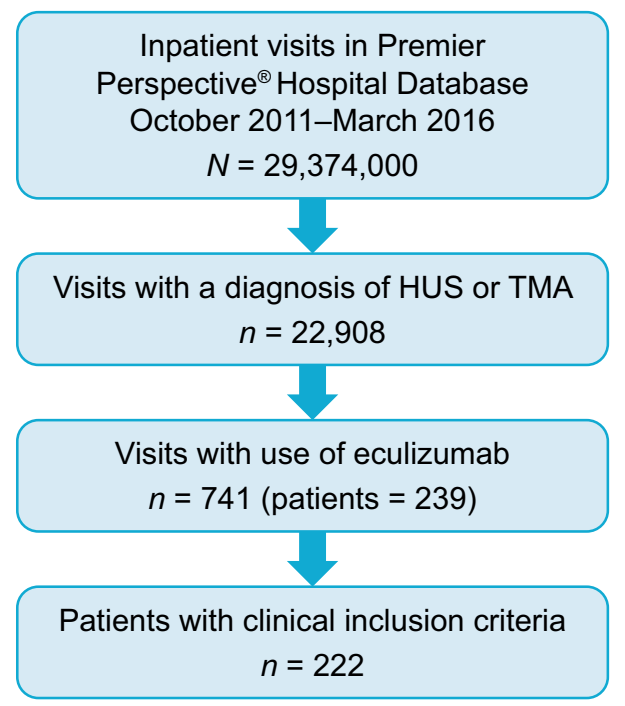

late initiators were readmitted within 90 days of initial hospitalisation $(36.7 \%[n=55 / 150]$ vs. $22.2 \%[n=16 / 72]$; $p=0.031)$. There was a numerically higher percentage of deaths with late initiation $(10.7 \%[n=16 / 150]$ vs. $6.9 \%$ $[n=5 / 72]$ ), although cause of death was not recorded. Mean time to discharge after eculizumab initiation was similar in the two groups (11.6 vs. 11.0 days), and most patients were discharged within 15 days (Fig. 1 in the ESM). The overall distribution of hospital costs is shown in Fig. 2 in the ESM. Late initiation was associated with significantly higher total hospital costs than early initiation (\$US143,580 vs. 87,386, respectively; $p=0.001$ ) (Table 2 ). Furthermore, significantly more late initiators received dialysis and plasmapheresis before starting eculizumab compared with early initiators, and late initiators spent significantly more time receiving plasmapheresis before starting eculizumab (Table 3 ).

In the multivariable regression analysis, late initiators were 3.2 times more likely to have dialysis during their hospital visit (95\% confidence interval 1.4-7.3) (Table 4). Estimated total hospital costs (least squares mean) were \$US103,557 in late initiators and \$US85,776 in early initiators $(p=0.0024)$. There were no differences between early and late initiators in time from first eculizumab dose to discharge and, unlike in the univariable analysis, there was no difference between the two groups in inpatient readmissions or deaths.

When the definition of early initiation of eculizumab was varied from $\leq 3$ to $\leq 10$ days, early initiation was significantly associated with reduced need for dialysis for all definitions and with reduced costs for all definitions of early initiation except $\leq 10$ days (Table 2 in the ESM). There was no significant association between early initiation and time to discharge, readmission, or death for any definition or early initiation.

Fig. 1 Patient flow. HUS haemolytic uraemic syndrome, TMA thrombotic microangiopathy

Table 1 Patient demographics

\begin{tabular}{lllc}
\hline Demographic & Early initiators $(n=72)$ & Late initiators $(n=150)$ & $p$ value \\
\hline Age, years & $34.1 \pm 22.9$ & $45.9 \pm 20.5$ & $<0.001$ \\
Median (Q1-Q3) & $32.5(12-56.5)$ & $48.5(31-62)$ & $<0.0001$ \\
$<18$ years & $23(31.9)$ & $16(10.7)$ & 0.0210 \\
$\geq 65$ years & $6(8.3)$ & $31(20.7)$ & 0.106 \\
Female & $42(58.3)$ & $104(69.3)$ & \\
Race & & & 0.122 \\
White & $49(68.1)$ & $92(61.3)$ & \\
Black & $9(12.5)$ & $36(24.0)$ & \\
Other & $14(19.4)$ & $22(14.7)$ & 0.0005 \\
Elixhauser comorbidity score & $4.2 \pm 2.2$ & $5.4 \pm 2.3$ & \\
\hline
\end{tabular}

Data are presented as mean \pm standard deviation or $n(\%)$ unless otherwise indicated. Univariate comparison for continuous variables was conducted using a $t$ test and, for categorical variables, a chi-squared test 
Table 2 Hospital visit characteristics (unadjusted)

\begin{tabular}{lllr}
\hline Characteristic & Early initiators $(n=72)$ & Late initiators $(n=150)$ & $p$ value \\
\hline Length of stay & $16.4 \pm 11.2$ & $29.0 \pm 21.5$ & $97(64.7)$ \\
ICU admissions & $48(66.7)$ & $12.4 \pm 10.7$ & $<0.001$ \\
ICU length of stay & $8.9 \pm 8.4$ & $11.6 \pm 13.7$ & \\
Time of discharge from day 1 of eculizumab (excluding & $11.0 \pm 11.4$ & & \\
$\quad$ deaths) & & $88(58.7)$ \\
Discharge destination & $51(70.8)$ & $18(12.0)$ \\
Home or self-care & $9(12.5)$ & $28(18.7)$ \\
Home health organisation & $7(9.7)$ & $16(10.7)$ \\
Skilled nursing or other facility & $5(6.9)$ & $55(36.7)$ \\
Death & $16(22.2)$ & $143,580 \pm 132,528$ \\
Readmission $\leq 90$ days of initial hospitalisation & $87,386 \pm 61,734$ & 0.227 \\
Total hospital cost, \$US & & 0.031 \\
\hline
\end{tabular}

Data are presented as mean \pm standard deviation or $n(\%)$ unless otherwise indicated. Univariate comparison for continuous variables was conducted using a $t$ test and, for categorical variables, a chi-squared test

ICU intensive care unit

Table 3 Procedures undertaken during hospital stay

\begin{tabular}{lccr}
\hline Procedure & $\begin{array}{l}\text { Early initiators } \\
(n=72)\end{array}$ & $\begin{array}{l}\text { Late initiators } \\
(n=150)\end{array}$ & $p$ value \\
\hline Plasmapheresis prior to eculizumab & $30(41.7)$ & $111(74.0)$ & $<0.001$ \\
Days of plasmapheresis prior to eculizumab & $2.9(1.6)$ & $8.4(7.2)$ & $<0.001$ \\
Dialysis during hospital stay & $53(73.6)$ & $128(85.3)$ & 0.035 \\
\hline
\end{tabular}

Data presented are presented as $n(\%)$ unless otherwise stated

Table 4 Multivariable regression analysis

\begin{tabular}{lll}
\hline Variable & $\begin{array}{l}\text { Late vs. early initiators } \\
\text { (LSM estimates and ORs) }\end{array}$ & $p$ value \\
\hline $\begin{array}{l}\text { First dose of eculizumab to } \\
\text { discharge, days }\end{array}$ & 5.6 vs. 4.2 & 0.2627 \\
Readmissions $^{\mathrm{b}}$ & $1.3(0.6-2.6)$ & 0.5347 \\
Dialysis $^{\mathrm{b}}$ & $3.2(1.4-7.3)$ & 0.0049 \\
Death $^{\mathrm{b}}$ & $0.8(0.3-2.7)$ & 0.7891 \\
Total hospital costs, \$US $^{\mathrm{a}}$ & 103,557 vs. 85,776 & 0.0024 \\
\hline
\end{tabular}

LSM least squares mean, $O R$ odds ratio

${ }^{a}$ Data presented as least squares mean estimates

${ }^{b}$ Data presented as odds ratio of late initiators/early initiators $(95 \%$ confidence interval)

\section{Discussion}

The results of this analysis indicate that hospitalised patients with aHUS who initiate eculizumab more than 7 days after admission have worse economic outcomes than those who initiate eculizumab within 7 days. These results are consistent with those from other research with eculizumab in patients with aHUS [9]. Notably, late initiators had a longer length of ICU stay and overall hospital stay than did early initiators. As a result of these differences between the two groups, late initiation incurred a significantly greater total hospital cost than early initiation. In multivariable analysis, the difference in hospital costs remained significant, late initiators having estimated total hospital costs (least squares mean) that were $\$$ US17,781 higher than those of early initiators. The multivariable regression analysis also confirmed that late initiators were three times more likely to require dialysis during their hospital stay than early initiators. A previous cost analysis of eculizumab therapy compared with plasma therapies for a recurrent episode of aHUS showed that the difference in cost between eculizumab and plasma therapies varied significantly according to the weight of the individual and the frequency of maintenance plasma therapy [16]. Furthermore, the additional cost related to 3 months of eculizumab treatment, particularly in patients weighing $>40 \mathrm{~kg}$, was similar to the cost saving accrued by rescuing a renal allograft from early failure. Overall, the authors of that study concluded that short-term eculizumab is the most cost-effective strategy to treat aHUS recurrence [16].

This paper further adds to the literature by confirming, in a real-world setting, the benefit of eculizumab therapy from 
a health resource utilisation perspective. The results of this analysis, together with previous studies showing reduced mortality rates in patients with aHUS receiving eculizumab [2], highlight the importance of an early diagnosis, for both saving lives and reducing hospital costs. The results also highlight the importance of multidisciplinary education focusing on specialities that are first-line treaters of TMA, including emergency room physicians. This educational dialogue would emphasise the importance of collecting blood samples before initiating plasma exchange to avoid compromising ADAMTS13 results. Differentiation among the causes of TMA should be made more quickly, based on ADAMTS13 testing and other criteria, to initiate timely and appropriate treatment. To facilitate this, education of physicians in interpretation of laboratory results is essential, and hospitals should have a single point of contact, whether an individual or committee, for expert advice. To expedite results of ADAMSTS13 test, when feasible, choosing testing facilities with rapid response time or moving the assay in-house is recommended. Based on the results of this analysis, we propose the following four action items for those concerned with the care of a patient with suspected TMA.

1. We propose education of health professionals that an early, accurate diagnosis is critical, not only in terms of reducing time on dialysis and improving outcomes but also in terms of total hospital costs.

2. We recommend testing for ADAMTS13 activity and inhibitors as soon as TMA is suspected. For larger hospitals and particularly tertiary care centres, we recommend moving ADAMTS13 testing in-house wherever possible to reduce the waiting times for results from $>4-7$ days to within 1 day $[10,11]$.

3. We recommend hospital plasmapheresis directors be educated as to the need to obtain ADAMTS13 testing, as the results of this test informs the necessity for plasma exchange or infusion, which are costly and may be unnecessary.

4. As is current practice at some institutions, we propose that larger hospitals have established TMA teams with physicians drawn from a variety of pertinent subspecialties, particularly haematology/oncology, nephrology, rheumatology and obstetrics, with expertise in the diagnosis of aHUS.

The main strength of this study is that it is the largest analysis conducted so far to address the issue of whether early initiation of eculizumab is associated with less resource use and lower costs compared with later initiation. It is possible that a different cut-off point for early versus late initiation might alter the results. The cut-off of 7 days was chosen based on data showing that initiation within 7 days was associated with improved eGFR compared with later initiation [9]. This cut-off also accounts for the time needed to receive ADAMTS13 results to rule out TTP. However, ideally, ADAMTS13 results should be available much more quickly to facilitate fast, appropriate management, as the test itself takes only a few hours. The main limitation is that because this was a relatively small sample, not all comorbidities could be added to the analysis, therefore a comorbidity (Elixhauser) score, based on 31 comorbidity categories (including congestive heart failure, hypertension, diabetes, renal failure, liver disease, metastatic cancer) was used as a surrogate. It is possible that several comorbidities serving as possible triggers for aHUS (e.g. autoimmune disease, pregnancy and cancer chemotherapy [17]) may have been confounding factors that could not be assessed in this study. Additionally, as this was a retrospective review of data from a resource utilization database, we cannot exclude the possibility of disease severity having an impact on the results. We assumed that disease severity was evenly distributed among early and late initiators of eculizumab, but we cannot rule out the possibility that more patients with severe disease could have been included in the early initiators group. The factors that contributed to early versus late initiation decisions were determined by the treating physician, and these data were not available in the database. Treatment decisions were not specifically reviewed, nor were medical charts as this was not the purpose of the analysis. Finally, this was a retrospective analysis of a claims database and, therefore, clinically important parameters (such as renal function, weight, etc.) are unavailable as the database is not designed for clinical research. Thus, information on patient characteristics is therefore somewhat limited.

\section{Conclusion}

Early initiators of eculizumab (within 7 days of hospital admission) had significantly lower rates of dialysis during their hospital stay than late initiators. Total hospital costs were significantly higher for late initiators than for early initiators, and they spent significantly more time in the ICU and received more plasmapheresis therapy before starting eculizumab than early initiators.

Acknowledgements Medical writing support was provided by Dr Daniel Booth, PhD, and Dr Ciaran Wright, PhD (Bioscript, Macclesfield, UK) and funded by Alexion Pharmaceuticals, Inc. All authors discussed and agreed the content, which derived from previous posters presented at scientific congresses. All authors reviewed and provided comprehensive feedback of all manuscript drafts and approved the final version for journal submission.

Data Availability Statement Patient-level data used in this study were obtained on a contractual basis from Premier Healthcare Database (https://www.premierinc.com/; https://products.premierinc.com/appli ed-sciences/solutions/data). The analysis plan was limited to results 
highlighted in this manuscript. The datasets generated are not publicly available because of their proprietary nature.

\section{Compliance with Ethical Standards}

Funding This study was funded by Alexion Pharmaceuticals, Inc. (study funder). Study design was undertaken by Bonnie M.K. Donato and Gilbert L'Italien (former employees of study funder). Study conduct was overseen by Michael Ryan and William Irish (contracted by study funder). Data analysis and interpretation was undertaken by Christoph Gasteyger (employee of study funder) and Jeffrey Laurence (a consultant to the study funder). The first draft of the manuscript was developed by the medical writer (contracted by the study funder) under guidance from the authors.

Conflict of interest Michael Ryan is an employee of CTI, a paid consultant of Alexion. Bonnie M.K. Donato is an employee of Boehringer-Ingelheim Pharmaceutical Inc. and was an employee of Alexion Pharmaceuticals, Inc. at the time of the study. William Irish has no disclosures to declare. Christoph Gasteyger is an employee of Alexion Pharma GmbH. Gilbert L'Italien is a former employee of Alexion Pharmaceuticals, Inc. Jeffrey Laurence is a consultant to Alexion.

Statement of human rights This study has been approved by the appropriate institutional and/or national research ethics committee and has been performed in accordance with the ethical standards as laid down in the 1964 Declaration of Helsinki and its later amendments.

Open Access This article is distributed under the terms of the Creative Commons Attribution-NonCommercial 4.0 International License (http://creativecommons.org/licenses/by-nc/4.0/), which permits any noncommercial use, distribution, and reproduction in any medium, provided you give appropriate credit to the original author(s) and the source, provide a link to the Creative Commons license, and indicate if changes were made.

\section{References}

1. Azoulay E, et al. Expert statements on the standard of care in critically ill adult patients with atypical hemolytic uremic syndrome. Chest. 2017;152(2):424-34.

2. Fakhouri F, Zuber J, Fremeaux-Bacchi V, Loirat C. Haemolytic uraemic syndrome. Lancet. 2017;390(10095):681-96.

3. Noris M, et al. Relative role of genetic complement abnormalities in sporadic and familial aHUS and their impact on clinical phenotype. Clin J Am Soc Nephrol. 2010;5:1844-59.
4. Fremeaux-Bacchi V, et al. Genetics and outcome of atypical hemolytic uremic syndrome: a nationwide French series comparing children and adults. Clin J Am Soc Nephrol. 2013;8(4):554-62.

5. Rother RP, Rollins SA, Mojcik CF, Brodsky RA, Bell L. Discovery and development of the complement inhibitor eculizumab for the treatment of paroxysmal nocturnal hemoglobinuria. Nat Biotechnol. 2007;25(11):1256-64.

6. Ueda Y, et al. Differential contribution of C5aR and C5b-9 pathways to renal thrombic microangiopathy and macrovascular thrombosis in mice carrying an atypical hemolytic syndromerelated factor $\mathrm{H}$ mutation. Kidney Int. 2019;96(1):67-79.

7. Legendre CM, et al. Terminal complement inhibitor eculizumab in atypical hemolytic-uremic syndrome. N Engl J Med. 2013;368(23):2169-81.

8. Licht $\mathrm{C}$, et al. Efficacy and safety of eculizumab in atypical hemolytic uremic syndrome from 2-year extensions of phase 2 studies. Kidney Int. 2015;87(5):1061-73.

9. Vande Walle J, Delmas Y, Ardissino G, Wang J, Kincaid JF, Haller $\mathrm{H}$. Improved renal recovery in patients with atypical hemolytic uremic syndrome following rapid initiation of eculizumab treatment. J Nephrol. 2017;30(1):127-34.

10. Laurence J. Atypical hemolytic uremic syndrome (aHUS): treating the patient. Clinical Adv Hematol Oncol. 2013;11(10, S15):4-15.

11. Connell NT, Cheves T, Sweeney JD. Effect of ADAMTS13 activity turnaround time on plasma utilization for suspect thrombotic thrombocytopenic purpura. Transfusion. 2016;56:354-9.

12. Laurence J, Haller H, Mannucci PM, Nangaku M, Praga M, Rodriguez de Cordoba S. Atypical hemolytic uremic syndrome (aHUS): essential aspects of an accurate diagnosis. Clin Adv Hematol Oncol. 2016;14(Suppl 11):2-15.

13. Zuber J, et al. Use of eculizumab for atypical haemolytic uraemic syndrome and C3 glomerulopathies. Nat Rev Nephrol. 2012;8:643-57.

14. Siedlecki AM, et al. Eculizumab use for kidney transplantation in patients with a diagnosis of atypical hemolytic uremic syndrome. Kidney Int Rep. 2019;4:434-46.

15. Elixhauser A, et al. Comorbidity measures for use with administrative data. Med Care. 1998;36:8-27.

16. Zuber J, et al. Eculizumab for atypical hemolytic uremic syndrome recurrence in renal transplantation. Am J Transplant. 2012;12(12):3337-54.

17. Schönermarck U, et al. Relative incidence of thrombotic thrombocytopenic purpura and haemolytic uraemic syndrome in clinically suspected cases of thrombotic microangiopathy. Clin Kidney J. 2019. https://doi.org/10.1093/ckj/sfz066. 